\title{
In Memory of Kenneth G. Wilson
}

\author{
Franz J. Wegner
}

Received: 17 March 2014 / Accepted: 27 March 2014 / Published online: 29 April 2014

C) Springer Science+Business Media New York 2014

\begin{abstract}
Kenneth Wilson had an enormous impact on the renormalization group and field theories in general. I had the great pleasure to work in three fields to which he contributed essentially: Critical phenomena, gauge-invariance in duality and confinement, and flow equations and similarity renormalization.
\end{abstract}

Keywords Renormalization group - Critical phenomena - Gauge-invariant models · Duality $\cdot$ Similarity transformation $\cdot$ Flow equations

\section{Introduction}

I am thankful, that several times Ken and I could work on similar problems. We owe him a lot in critical phenomena, where he developed a calculational scheme to determine critical exponents and scaling functions on the basis of renormalization group ideas (Sect. 2).

A second time I came close to his work. I had generalized the Kramers-Wannier duality of the two-dimensional Ising model to higher-dimensional models. This procedure yields gauge-invariant models. Wilson generalized these ideas to non-abelian gauge theories and developed a theory for the confinement of quarks (Sect. 3).

In 1993/94 Głazek and Wilson and independently the present author developed the idea of a canonical transformation which diagonalizes a many-particle hamiltonian (Sect. 4).

I will shortly report on these developments and imbed them in the state the field had reached before and add some results grown out of these developments. I apologize to all I do not mention in this article. There would be simply too many to be cited.

\footnotetext{
F. J. Wegner $(\varangle)$

Institute for Theoretical Physics, Ruprecht-Karls-University,

Heidelberg, Germany

e-mail: wegner@tphys.uni-heidelberg.de
} 


\section{Renormalization Group and Critical Phenomena}

I have seen Ken Wilson for the first time at the 1970 Mid-winter solid-state research conference at Newport Beach [45]. It was my first trip to the United States. I was delighted to meet all the scientists working in critical phenomena, whom I knew from their papers.

What was known before Wilson presented his renormalization group ideas on critical phenomena in 1971? Initially critical phenomena were only described by molecular field type theories. The oldest one is due to Van der Waals [63] in 1873 for the gas-liquid transition. Curie [11] and Weiss [77] formulated it for ferromagnets, and Landau [43,44] for general systems. They all predicted that the order parameter behaves below $T_{c}$ like $\propto\left(T_{c}-T\right)^{\beta}$ with $\beta=1 / 2$. However already in 1900 Verschaffelt [66] observed $\beta=0.34$.. for the difference of the density of the liquid and the vapor of isopentan, where he had analyzed data by Young [89]. He also found along the isotherm $\rho-\rho_{c} \propto\left(P-P_{c}\right)^{1 / \delta}$ with $\delta=4.259$ instead of $\delta=3$ by Van der Waals. Later on many other experiments on gas-fluid systems, ferromagnets and other systems gave exponents different from molecular type ones. Also the specific heat often did not show a jump as predicted by molecular field theory, but a divergency or cusp like $\left|T-T_{c}\right|^{-\alpha}$ or $-\ln \left|T-T_{c}\right|$. Levelt-Sengers [46] has given a useful review on the history of critical phenomena. Two models showed deviations from molecular field behaviour: The twodimensional Ising model (Onsager [52]) gave a logarithmic singularity of the specific heat and a critical exponent $\beta=1 / 8$ for the spontaneous magnetization [88]. Berlin and Kac [4] developed the spherical model, which below dimension 4 showed partly a different behaviour. For the three-dimensional model one had still $\beta=1 / 2$, but a kink in the specific heat, $\alpha=-1$. The susceptibility $\chi \propto\left(T-T_{c}\right)^{-\gamma}$ yields $\gamma=2$ in this model in contrast to the molecular field value $\gamma=1$. If the dimension $d$ of the system is considered a continuous variable, then this model shows clearly that the exponents depend on the dimensionality between $d=2$ and $d=4$. Above four dimensions this model shows molecular field behaviour. Ginzburg [25] has argued that in three-dimensional systems there is a temperature region, in which molecular field-theory fails. Extending his arguments to higher dimensions one finds that only above four dimensions molecular field theory is correct.

Inequalities for critical exponents were established [21,26,27,57]. Widom [80] had the beautiful idea that the difference of various thermodynamic quantities from their critical values, e.g. that of the chemical potential, density, and temperature in a fluid, are related by homogeneous functions. This explains equalities between critical exponents and also equal exponents above and below the critical temperature Compare Griffiths [28]. Such relations between exponents had been guessed before. This homogeneity picture was confirmed by Kadanoff's cell model [40]. Using his own words: The description is based upon dividing the Ising model into cells which are microscopically large but much smaller than the coherence length and then using the total magnetization within each cell as a collective variable. In this way he considered the effective interaction on different length scales. He introduces the mapping of the interaction of the original spins to the new cell spins. The critical point is related to the fixed point of this mapping. Small deviations from the fixed-point interaction grow (or decay) with certain factors from one length scale to the other. This allows the singular part of the free energy to be written as a homogeneous function in the sense of Widom.

The experimental observation that real systems are not described by molecular field theory were supported by the calculation and analysis of series expansions, in particular of hightemperature expansions. These expansions gave exponents not very far from measured ones. A good review is volume 3 [16] of the Domb-Green series. These estimates led to the hypothesis of universality as for example expressed by Fisher et al. [22,29,39]. Accordingly the exponents depend only on the dimension $d$ of the system, the dimension $n$ of the (easy 
components of the) order parameter, and on the range of interaction, if it decreases slowly with distance. Watson [67] even argued that certain ratios of amplitudes did not depend on the lattice of the model but only on the universality class. Soon later two groups introduced the idea of universality classes to critical dynamics: Ferrell et al. $[18,19]$ as well as Halperin and Hohenberg who carried over the ideas of Widom and Kadanoff to critical dynamics [31,32].

The calculations of critical exponents in isotropic and anisotropic classical Heisenberg models by Jasnow and Wortis [39] inspired Eberhard Riedel and myself to derive homogeneity relations for this model [55], where we introduced the crossover exponent and argued how the temperature region showing anisotropic behaviour shrinks with the anisotropy. I had done some work on magnetic ordering [68,69] and on critical spin dynamics [70,71]. In the group of Wilhelm Brenig and Herbert Wagner in Munich we discussed Kadanoff's cell model [40] and the reviews by Fisher [23], Leo Kadanoff et al. [41], and by Heller [34]. Therefore I knew the fascinating hypotheses and results in critical phenomena and the open problems. In 1971 I spent a year as postdoc in the group of Leo Kadanoff. Although at that time Leo worked on urban problems, he was much interested in critical phenomena, and I had useful discussions with him. We wrote a paper [42] in connection with the solution of the eight-vertex-model, which had just been solved by Baxter [3].

In 1971 Wilson's two seminal papers [81,82] on the critical behavior of the Ising model appeared. I became really aware of them, when Ken Wilson's and Michael Fisher's paper [86] on the $4-\epsilon$ expansion of critical exponents of the $n$-vector model appeared. I was fascinated that here was a theory which easily fulfilled the Ginzburg criterion [25]. From then on I worked on $4-\epsilon$-expansions $[30,73,76]$, partially with colleagues at Brown University, and I investigated general consequences of the Kadanoff-Wilson renormalization group picture $[72,74]$. Much of this work as well as that of other scientists is reported in volume 6 of the Domb-Green series [16], which after Melville Green's death was continued by Cyril Domb and Joel Lebowitz [17]. Wilson's work gave a clear understanding of critical phenomena and simultaneously the possibility for explicit calculations starting from the upper critical dimension four. Once I visited at Cornell and I remember a lively discussion with Ken Wilson and Michael Fisher on the $\epsilon$-expansion for the cross-over exponent [24,73]. Shortly later Eberhard Riedel and myself investigated the tricritical point $[56,77]$ with upper critical dimension $d=3$.

Stanley [59] had shown that the $n=\infty$-limit of the $n$-vector model is described by the spherical model. It did not take long until $1 / n$-expansions for the $n$-component spin model attracted Abe [1], Ma [47], Suzuki [61], Ferrell and Scalapino [20], and also Wilson [84]. Shang-keng Ma gave a review [48]. An elobarate technique was later given by Vasil'ev et al. $[64,65]$.

Wilson's early papers on this subject and the review by Wilson and Kogut [87] integrated over the short wavelength components of the order parameter in the Lagrangian. Very fast it became clear, that the Landau theory of phase transitions $[43,44]$, which at first glance gave only molecular field exponents is very useful as field theory and became a basic theory for critical phenomena. Wilson [83] and Brézin et al. [7] applied Feynman-graph techniques to this theory and one switched to dimensional regularization. See the review by Brézin et al. [10]. Similarly di Castro and Jona-Lasinio showed that the multiplicative renormalization group provides scaling laws [13] and critical exponents from the $\epsilon$-expansion [12]. However, in many cases functional renormalization is very useful $[37,51,53,62,79,87]$. Wilson's description of critical phenomena was soon applied to critical dynamics. Halperin, Hohenberg, and Ma derived the consequences for the kinetic Ising model and showed the improvement over previous techniques [33]. A summary of the various dynamical universality classes is given in the review [36]. Some other aspects of critical phenomena are finite-size 
scaling (review by Barber) [2], critical surfaces and interfaces (reviews by Binder, Diehl and Jasnow) $[6,14,38]$, and wetting (review by Dietrich) [15].

Mermin and Wagner [49] had shown that continuous symmetries cannot be broken in $d \leq 2$ dimensions at finite temperatures provided the interaction is not too long-range. Migdal [50] and Polyakov [54] started an expansion for critical exponents from this lower dimensionality two, work which has been continued by e.g. Brézin et al. [8,9,35]. I became much interested in this expansion, since Lothar Schäfer and myself [58,75] could map the mobility edge problem of the Anderson model of particles on disordered lattices on a matrix-model, which can be investigated in $2+\epsilon$ dimensions [5].

It may be remarked that Stanley has compiled many papers on critical phenomena before and at the beginning of the Wilson era in a bibliography [60].

Wilson gave a beautiful review of the use of the renormalization group in critical pheneomena and he solved the s-wave Kondo Hamiltonian by a nondiagrammatic computer method [85].

Wilson's work in critical phenomena had an enormous impact on this field, since simultaneously it allowed explicite calculations and gave an intuitive picture of the renormalization group procedure.

\section{Duality and Confinement}

In 1970-1971 I thought about duality of Ising-like models in dimensions larger than two, similar to the Kramers-Wannier duality [94] of the two-dimensional Ising model. It occured to me that such dual models could be expressed as gauge-invariant Ising-models, which may also show a transition, but without a local order parameter [96]. In four dimension a gaugeinvariant model became self-dual and had the same transition temperature as the ordinary two-dimensional Ising-model. Instead of a local order-parameter the product of spins along a closed loop showed a different behavior in both phases. At high temperature it obeyed an area law, at low temperatures a perimeter law,

$$
\left\langle\prod_{i \in \text { loop }} S_{i}\right\rangle= \begin{cases}\exp (-a / \alpha(T)) & T>T_{\mathrm{c}} \\ \exp (-l / \xi(T)) & T<T_{\mathrm{c}}\end{cases}
$$

where $a$ is the enclosed area and $l$ the length of the perimeter.

Ken Wilson generalized this idea to continuous gauge-theories [97], which allowed him to describe the confinement of quarks, a description, which became very important in highenergy physics. Other important contributions were by Balian et al. [90-92].

The transition temperature for the Ising gauge model was confirmed by Creutz et al. [93] numerically to the precision allowed by hysteresis effects at the first-order transition. Many papers of this subject are compiled in a review volume by Rebbi [95].

\section{Flow Equations and Similarity Renormalization}

Our work came very close for a third time. Głazek and Wilson $[123,124](\mathrm{GW})$ and myself [158] (W) developed independently equations for a unitary hamiltonian flow, which brings the hamiltonian in diagonal or block-diagonal form. It is also called similarity transformation or similarity renormalization. Common to both procedures is that one approaches the diagonal form continuously. This may be written 


$$
\frac{\mathrm{d} H(\ell)}{\mathrm{d} \ell}=[\eta(\ell), H(\ell)]
$$

with the generator $\eta(\ell)$ of the unitary transformation and $\ell$ the flow-parameter. One starts with $H(0)=H$ and reaches diagonalization or block-diagonalization in the limit $\ell \rightarrow \infty$.

The main difference is that $(\mathrm{GW})$ in their original formulation eliminate the off-diagonal matrix elements between states of an energy difference larger than some $\Delta E$ completely, which shrinks continuously during the flow, whereas (W) suggests a smoother elimination procedure, given by

$$
\eta(\ell)=\left[H_{\mathrm{d}}(\ell), H(\ell)\right]
$$

where $H_{\mathrm{d}}$ is the diagonal part of the Hamiltonian or some other appropriate $H_{\mathrm{d}}$ derived from $H$. (GW) working in high-energy physics take care of the renormalization of ultraviolett divergencies, whereas (W) starts from solid-state models on a lattice, where such divergencies no longer show up. Both procedures aim at the infrared problem. These methods are apt for fermionic and bosonic and even spin models.

Later it was realized that the mathematicians Brockett et al. [111-113] performed similar transformations in information theory. They call the method double bracket flow and isospectral flow, resp. The notion double bracket flow becomes obvious, when one inserts (3) in (2). The two-beam coupling in photorefractive media itself obeys the flow equation scheme [98].

It is special for fermionic solid-state systems that the important physics takes place at the Fermi edge which in dimension $d>1$ is no longer restricted to one or two points in momentum space, but extends over a $d-1$ dimensional region. Thus Shankar $[153,154]$ eliminates states away from the Fermi edge and keeps only those very close to it. Similar elimination ideas not for the Hamiltonian, but for irreducible vertices in the form of Polchinski equations [53] were introduced by Zanchi and Schulz [162], Halboth and Metzner [130], and Salmhofer and Honerkamp [151].

The flow equation scheme was applied in many cases in high-energy physics, nuclear physics and atomic physics. I mention some work in light front QED [128,133] and in light front QCD [110,120,161] and in effective nuclear interactions [104,105] and for nuclear few body problems $[102,103]$.

Głazek and Wilson $[126,127]$ investigated the possibility of limit cycles. The connection between asymptotic freedom and limit cycles has been studied in [121,122]. Already in 1970 Ken Wilson was interested in systems invariant under a change of energy scale [160]. Consequently Głazek and Wilson investigated systems whose states had energies and interactions increasing by powers of some factors $b>1$ [121,125]. An infrared limit cycle in QCD was suggested by Braaten and Hammer [106]. A nice example for limit cycles [99-101,149] are the Efimov states [114,115,156], first investigated for helium3 and tritium. Such states became of interest in the physics of ultracold atoms [107], when they are tuned to large scattering lengths [143] close to the Feshbach resonance [118].

There are numerous applications in solid-state physics. The smoothness of the transformation yields smoother results for the elimination of the electron-phonon interaction in superconductors [147,148] than the transformation by Fröhlich [119]. The flow equation result comes close to those by Eliashberg [116,117], but in a simpler way. Similar smooth results are obtained for the Anderson impurity model [137] in contrast to the Schrieffer-Wolf transformation [152]. A model closely related to the mechanism of dissipation is the spinboson model, which in this framework was first treated by Kehrein et al. [138-141]. Various aspects of the Kondo model can be investigated by this method [132,135,155,157]. Also the dynamics of spins on certain lattices can be investigated [108,109,142,150]. 
Electronic systems in $d>1$ dimensions can be brought to the form described by Landau's Fermi liquid theory [144-146] by means of flow equations [134,136]. The Hubbard model was treated in the weak-coupling limit $[129,131]$, where the Hamitonian was not diagonalized, but brought to a block-diagonal form, for which molecular field theory is exact. Thus various instabilities (antiferromagnetism, d-wave-superconductivity, Pomeranchuk instability) can be read from this block-diagonal form. Many applications in solid state theory can be found in the short review [159] and in the book by Kehrein [136].

\section{Concluding Remark}

Ken Wilson's work in field theory and renormalization had an enormous impact. He was honored by the Nobel prize. Wilson also was engaged in Physics education. I am glad to have met Ken Wilson.

Acknowledgments I am indebted to Stan Głazek for providing me with a number of useful comments.

\section{References}

\section{Critical Phenomena and Renormalization Group}

1. Abe, R.: Expansion of a critical exponent in inverse powers of spin dimensionality. Prog. Theor. Phys. 48, 1414 (1972)

2. Barber, M.N.: Finite size scaling, in [16], 8, 145 (1983)

3. Baxter, R.J.: Eight-vertex model in lattice statistics. Phys. Rev. Lett. 26, 832 (1971)

4. Berlin, T.H., Kac, M.: The spherical model of a ferromagnet. Phys. Rev. 86, 821 (1952)

5. Bernreuther, W., Wegner, F.J.: Four-loop order $\beta$-function for two dimensional non-linear $\sigma$-models. Phys. Rev. Lett. 57, 1383 (1986)

6. Binder, K.: Critical behavior at surfaces in [16], 8, 1 (1983)

7. Brézin, E., Wallace, D.J., Wilson, K.G.: Feynman-graph expansion for the equation of state near the critical point (Ising-like case). Phys. Rev. Lett. 29, 591 (1972)

8. Brézin, E., Zinn-Justin, J.: Renormalization of the nonlinear $\sigma$ model in $2+\epsilon$ dimensions - application to the Heisenberg ferromagnet. Phys. Rev. Lett. 36, 691 (1976)

9. Brézin, E., Zinn-Justin, J.: Spontaneous breakdown of continuous symmetries near two dimensions. Phys. Rev. B14, 3110 (1976)

10. Brézin, E., LeGuillou, J.C., Zinn-Justin, J.: Field theoretical approach to critical phenomena in [15], 6, 126 (1976)

11. Curie, P.: Propriétés magnétiques des corps á diverses températures (Magnetic properties of bodies at different temperatures). Dissertation, Paris (1895)

12. di Castro, C.: The multiplicative renormalization group and the critical behavior in $d=4-\epsilon$ dimensions. Lett. Nuov. Cim. 5, 69 (1972)

13. di Castro, C., Jona-Lasinio, G.: On the microscopic foundation of scaling laws. Phys. Lett. A29, 322 (1969)

14. Diehl, H.W.: Field-theoretic approach to critical behavior at surfaces, in [16] 10, 75 (1986)

15. Dietrich, S.: Wetting phenomena, in [16] 12, 1 (1988)

16. Domb, C., Green, M.S. (eds) Phase Transitions and Critical Phenomena, vols. 1-6. Academic Press, New York

17. Domb, C., Lebowitz, J.L. (eds.) Phase Transitions and Critical Phenomena, vols. 7-20. Academic Press, New York

18. Ferrell, R.A., Menyhárd, N., Schmidt, H., Schwabl, F., Szépfalusy, P.: Dispersion in second sound and anomalous heat conduction at the lambda point of liquid helium. Phys. Rev. Lett. 18, 891 (1967)

19. Ferrell, R.A., Menyhárd, N., Schmidt, H., Schwabl, F., Szépfalusy, P.: Fluctuations and lambda phase transition in liquid helium. Ann. Phys. (NY) 47, 565 (1968) 
20. Ferrell, R.A., Scalapino, D.J.: Order parameter correlations within the screening approximation. Phys. Rev. Lett. 29, 413 (1972)

21. Fisher, M.E.: Correlation functions and the critical region of simple fluids. J. Math. Phys. 5, 944 (1964)

22. Fisher, M.E.: Quantum corrections to critical-point behavior. Phys. Rev. Lett. 16, 11 (1966)

23. Fisher, M.E.: The theory of critical phenomena. Rep. Prog. Phys. 30, 615 (1967)

24. Fisher, M.E., Pfeuty, P.: Critical behavior of the anisotropic $n$-vector model. Phys. Rev. B6, 1889 (1972)

25. Ginzburg, V.L.: Some remarks on phase transitions of the second kind and the microscopic theory of ferroelectric materials. Sov. Phys. Solid State 2, 1824 (1960)

26. Griffiths, R.B.: Thermodynamic inequality near the critical point for ferromagnets and fluids. Phys. Rev. Lett. 14, 623 (1965)

27. Griffiths, R.B.: Ferromagnets and simple fluids near the critical point: some thermodynamic inequalities. J. Chem. Phys. 43, 1958 (1965)

28. Griffiths, R.B.: Thermodynamic functions for fluids and ferromagnets near the critical point. Phys. Rev. 158, 176 (1967)

29. Griffiths, R.B.: Dependence of critical indices on a parameter. Phys. Rev. Lett. 24, 1479 (1970)

30. Grover, M.K., Kadanoff, L.P., Wegner, F.J.: Critical exponents for the Heisenberg model. Phys. Rev. B6, 311 (1972)

31. Halperin, B.I., Hohenberg, P.C.: Generalization of scaling laws to dynamical properties of a system near its critical point. Phys. Rev. Lett. 19, 700 (1967)

32. Halperin, B.I., Hohenberg, P.C.: Scaling laws for dynamic critical phenomena. Phys. Rev. 177, 952 (1969)

33. Halperin, B.I., Hohenberg, P.C., Ma, S.-K.: Calculation of dynamical critical properties using Wilson's expansion method. Phys. Rev. Lett. 29, 1548 (1972)

34. Heller, P.: Experimental investigations of critical phenomena. Rep. Prog. Phys. 30, 731 (1967)

35. Hikami, S., Brézin, E.: Three-loop calculations in the two-dimensional non-linear $\sigma$-model. J. Phys. A: Math. Gen. 11, 1141 (1978)

36. Hohenberg, P.C., Halperin, B.I.: Theory of dynamical critical phenomena. Rev. Mod. Phys. 49, 435 (1977)

37. Houghton, A., Wegner, F.J.: Renormalization group equation for critical phenomena. Phys. Rev. A8, 401 (1973)

38. Jasnow, D.: Renormalization group of interfaces, in [16] 10, 269 (1986)

39. Jasnow, D., Wortis, M.: High temperature critical indices for the classical anisotropic Heisenberg model. Phys. Rev. 176, 739 (1968)

40. Kadanoff, L.P.: Scaling laws for Ising models near $T_{c}$. Physics 2, 263 (1966)

41. Kadanoff, L.P., Götze, W., Hamblen, D., Hecht, R., Lewis, E.A.S., Palciauskas, V.V., Rayl, M., Swift, J., Aspnes, D., Kane, J.: Static phenomena near critical points: theory and experiment. Rev. Mod. Phys. 39, 395 (1967)

42. Kadanoff, L.P., Wegner, F.J.: Some critical properties of the eight-vertex model. Phys. Rev. B4, 3989 (1971)

43. Landau, L.D.: On the theory of phase transformations. I. Phys. Z. Sowjet. 11, 26 (1937)

44. Landau, L.D.: On the theory of phase transitions. JETP 7, 19 (1937)

45. Laramore, G.E.: A report on the midwinter solid-state research conference. J. Stat. Phys. 2, 107 (1970)

46. Levelt-Sengers, J.M.H.: From Van der Waals' equation to the scaling laws. Physica 73, 73 (1974)

47. Ma, S.-K.: Critical exponents for charged and neutral gases above $\lambda$ points. Phys. Rev. Lett. 29, 1311 (1972)

48. Ma, S.-K.: The $1 / n$ expansion in [15], 6, 249 (1976)

49. Mermin, N.D., Wagner, H.: Absence of ferromagnetism or antiferromagnetism in one- or twodimensional isotropic Heisenberg models. Phys. Rev. Lett. 17, 1133 (1966)

50. Migdal, A.A.: Phase transitions in gauge and spin-lattice systems. Zh. Eksp. Teor. Fiz. 69, 1457; JETP 42, 743 (1975)

51. Morris, T.: The exact renormalization group and approximate solutions. Int. J. Mod. Phys. A9, 2411 (1994)

52. Onsager, L.: Crystal statistics. I. A two-dimensional model with an order-disorder transition. Phys. Rev. 65, 117 (1944)

53. Polchinski, J.: Renormalization and effective Lagrangians. Nucl. Phys. B 231, 269 (1984)

54. Polyakov, A.M.: Interaction of Goldstone particles in two dimensions. Applications to ferromagnets and massive Yang-Mills fields. Phys. Lett. 59B, 79 (1975)

55. Riedel, E., Wegner, F.: Scaling approach to anisotropic magnetic systems, statics. Z. Phys. 225, 195 (1969)

56. Riedel, E.K., Wegner, F.J.: Tricritical exponents and scaling fields. Phys. Rev. lett. 29, 349 (1972) 
57. Rushbrooke, G.S.: On the thermodynamics of the critical region for the Ising problem. J. Chem. Phys. 39, 842 (1963)

58. Schäfer, L., Wegner, F.: Disordered system with $n$ orbitals per site: Lagrange formulation, hyperbolic symmetry, and Goldstone modes. Z. Phys. B38, 113 (1980)

59. Stanley, H.E.: Spherical model as the limit of infinite spin dimensionalily. Phys. Rev. 176, 718 (1968)

60. Stanley, H.E.: Cooperative phenomena near phase transitions. A bibliography with selected readings. MIT Press, Cambridge (1973)

61. Suzuki, M.: Critical exponents and scaling relations for the classical vector model with long-range interactions. Phys. Lett. 42A, 5 (1972)

62. Tetradis, N., Wetterich, C.: Critical exponents from the effective action. Nucl. Phys. B422, 541 (1994)

63. Van der Waals, J.D.: Over de continuiteit van den gas- en vloeistoftoestand (On the continuity of the gaseous and the liquid state). Dissertation, Leiden (1873)

64. Vasil'ev, A.N., Pis'mak, Yu.M., Khonkonen, Yu.R.: Simple method of calculating the critical indices in 1/n expansion, Teor. Mat. Fiz. 46, 167 (1981). Theor. Math. Phys. 46, 104 (1981)

65. Vasil'ev, A.N., Pis'mak, Yu.M., Khonkonen, Yu.R.: $1 / n$ expansion: calculation of the exponent $v$ in the order $1 / n^{3}$ by the conformal bootstrap method. Teor. Mat. Fiz. 50, 195 (1982). Theor. Math. Phys. 50, 127 (1982)

66. Verschaffelt, J.E.: On the critical isothermal line and the density of saturated vapour and liquid in isopentane and carbone dioxide. Proc. Kon. Akad. Amst. 588 (1900); Commun. Phys. Lab. Leiden 55 (1900)

67. Watson, P.G.: Formation of invariants from critical amplitudes of ferromagnets. J. Phys. C2, 1883 (1969)

68. Wegner, F.: Magnetic ordering in one and two dimensional systems. Phys. lett. 24A, 131 (1967)

69. Wegner, F.: Spin-ordering in a planar classical Heisenberg model. Z. Phys. 206, 465 (1967)

70. Wegner, F.: On the Heisenberg model in the paramagnetic region and the critical point. Z. Phys. 216, 433 (1968)

71. Wegner, F.: On the dynamics of the Heisenberg antiferromagnet at $T_{N}$. Z. Phys. 218, 260 (1969)

72. Wegner, F.J.: Corrections to scaling laws. Phys. Rev. B5, 4529 (1972)

73. Wegner, F.J.: Critical exponents in isotropic spin systems. Phys. Rev. B6, 1891 (1972)

74. Wegner, F.J.: The critical state, general aspects in [15] 6, 7 (1976)

75. Wegner, F.: The mobility edge problem: continuous symmetry and a conjecture. Z. Phys. B35, 207 (1979)

76. Wegner, F.J., Houghton, A.: Feynman-graph calculation of the $(0, l)$ critical exponents to order $\epsilon^{2}$. Phys. Rev. A10, 435 (1974)

77. Wegner, F.J., Riedel, E.K.: Logarithmic corrections to the molecular-field behavior of critical and tricritical systems. Phys. Rev. B7, 248 (1973)

78. Weiss, P.: L'hypothése du champ moléculaire et la propriété ferromagnétique (Hypothesis of the molecular field and the ferromagnetic property). J. Phys. Theor. Appl. 6, 661 (1907)

79. Wetterich, C.: Exact evolution equation for the effective potential. Phys. Lett. B 301, 90 (1993)

80. Widom, B.: Equation of State in the neighborhood of the critical point. J. Chem. Phys 43, 3898 (1965)

81. Wilson, K.G.: Renormalization group and critical phenomena. I. Renormalization group and the Kadanoff scaling picture. Phys. Rev. B4, 3174 (1971)

82. Wilson, K.G.: Renormalization group and critical phenomena. II. Phase-space cell analysis of critical behavior. Phys. Rev. B4, 3184 (1971)

83. Wilson, K.G.: Feynman-graph expansion for critical exponents. Phys. Rev. Lett. 28, 548 (1972)

84. Wilson, K.G.: Quantum-field theory models in less than 4 dimensions. Phys. Rev. D7, 2911 (1973)

85. Wilson, K.G.: The renormalization group: critical phenomena and the Kondo problem. Rev. Mod. Phys. 47, 773 (1975)

86. Wilson, K.G., Fisher, M.E.: Critical exponents in 3.99 dimensions. Phys. Rev. Lett. 28, 240 (1972)

87. Wilson, K.G., Kogut, J.: The renormalization group and the $\epsilon$ expansion. Phys. Rep. 12C, 75 (1974)

88. Yang, C.N.: The spontaneous magnetization of a two-dimensional Ising model. Phys. Rev. 85, 808 (1952)

89. Young, S.: On the generalizations of Van der Waals regarding 'corresponding' temperatures, pressures, and volumes, Phil. Mag. 33, 153 (1892)

\section{Lattice Gauge Theories and Quark Confinement}

90. Balian, R., Drouffe, J.M., Itzykson, C.: Gauge fields on a lattice. I. General outlook. Phys. Rev. D10, 3376 (1974) 
91. Balian, R., Drouffe, J.M., Itzykson, C.: Gauge fields on a lattice. II. Gauge-invariant Ising model. Phys. Rev. D11, 2098 (1975)

92. Balian, R., Drouffe, J.M., Itzykson, C.: Gauge fields on a lattice. III. Strong-coupling expansions and transition points. Phys. Rev. D11, 2104 (1975)

93. Creutz, M., Jacobs, L., Rebbi, C.: Experiments with a gauge-invariant Ising system. Phys. Rev. Lett. 42, 1390 (1979)

94. Kramers, H.A.,Wannier, G.H.: Statistics of the two-dimensional ferromagnet. Part I. Phys. Rev. 60, 252 (1941)

95. Rebbi, C.: Lattice Gauge Theories and Monte Carlo Simulations. World Scientific, Singapore (1983)

96. Wegner, F.J.: Duality in generalized Ising models and phase transitions without local order parameter. J. Math. Phys. 12, 2259 (1971)

97. Wilson, K.G.: Confinement of quarks. Phys. Rev. D10, 2445 (1974)

\section{Flow Equations and Similarity Renormalization}

98. Anderson, D.Z., Brockett, R.W., Nutall, N.: Information dynamics of photorefractive two-beam coupling. Phys. Rev. Lett. 82, 1418 (1999)

99. Bedaque, P.F., Hammer, H.-W., van Kolck, U.: Renormalization of the three-body system with short range interactions. Phys. Rev. Lett. 82, 463 (1999)

100. Bedaque, P.F., Hammer, H.-W., van Kolck, U.: The three-boson system with short-range interactions. Nucl. Phys. A646, 444 (1999)

101. Bedaque, P.F., Hammer, H.-W., van Kolck, U.: Effective theory of the triton. Nucl. Phys. A676, 357 (2000)

102. Bogner, S.K., Furnstahl, R.J., Perry, R.J.: Similarity renoemalization group for nucleon-nucleon interactions. Phys. Rev. C75, 061001 (2007)

103. Bogner, S.K., Furnstahl, R.J., Perry, R.J., Schwenk, A.: Are low-energy nuclear observables sensitive to high-energy phase shifts? Phys. Lett. B649, 488 (2007)

104. Bogner, S.K., Schwenk, A., Kuo, T.T.S., Brown, G.E.: Renormalization group equation for low momentum effective nuclear interactions, arXiv:nucl-th/0111042 (2001)

105. Bogner, S.K., Kuo, T.T.S., Schwenk, A.: Model-independent low momentum nucleon interaction from phase shift equivalence. Phys. Rep. 386, 1 (2003)

106. Braaten, E., Hammer, H.-W.: An infrared renormalization group limit cycle in QCD. Phys. Rev. Lett. 91, 102002 (2003)

107. Braaten, E., Hammer, H.-W.: Efimov physics in cold atoms. Ann. Phys. (NY) 322, 120 (2007)

108. Brenig, W.: Spin dynamics of a tetrahedral cluster magnet. Phys. Rev. B67, 064402 (2003)

109. Brenig, W., Honecker, A.: Planar pyrochlore: a strong coupling analysis. Phys. Rev. B65, 140407 (2002)

110. Brisudova, M., Perry, R.: Initial bound state studies in light-front QCD. Phys. Rev. D54, 1831 (1996)

111. Brockett, R.W.: Dynamical systems that sort lists, diagonalize matrices, and solve linear programming problems. Linear Algebra Appl. 146, 79 (1991)

112. Chu, M.T.: A list of matrix flows with applications. Fields Inst. Commun. 3, 87 (1994)

113. Chu, M.T., Driessel, K.R.: The projected gradient method for least square matrix approximations with spectral constraints. SIAM J. Numer. Anal. 27, 1050 (1990)

114. Efimov, V.N.: Energy levels arising from resonant two-body forces in a three-body system. Phys. Lett. B33, 563 (1970)

115. Efimov, V.N.: Weakly-bound states of three resonantly interacting particles, Yad. Fiz. 12, 1080 (1970). Sov. J. Nucl. Phys. 12, 589 (1971)

116. Eliashberg, G.M.: Interactions between electrons and lattice vibrations, Zh. Eksp. Teor. Fiz. 28, 966, 1960; 29, 1437; Sov. Phys. JETP 11, 696 (1960)

117. Eliashberg, G.M.: Temperature Green's function for electrons in a superconductor. Zh. Eksp. Teor. Fiz. 29, 1437 (1960); Sov. Phys. JETP 12, 1000 (1960)

118. Feshbach, H.: Unified theory of nuclear rections. Ann. Phys. (NY) 5, 357 (1958)

119. Fröhlich, H.: Interaction of electrons with lattice vibrations. Proc. R. Soc. A215, 291 (1952)

120. Głazek, S.D.: Dynamics of effective gluons. Phys. Rev. D63, 116006 (2001)

121. Głazek, S.D.: Limit cycles of effective theories. Phys. Rev. D 75, 025005 (2007)

122. Głazek, S.D., Perry, R.J.: Impact of bound states on similarity renormalization group transformations. Phys. Rev. D 78, 045011 (2008)

123. Głazek, S.D., Wilson, K.G.: Renormalization of hamiltonians. Phys. Rev. D48, 5863 (1993) 
124. Głazek, S.D., Wilson, K.G.: Perturbative renormalization group for hamiltonians. Phys. Rev. D49, 4214 (1994)

125. Głazek, S.D., Wilson, K.G.: Asymptotic freedom and bound states in Hamiltonian dynamics. Phys. Rev. D57, 3558 (1998)

126. Głazek, S.D., Wilson, K.G.: Limit cycles in quantum theories. Phys. Rev. Lett. 89, 230401 (2002)

127. Głazek, S.D., Wilson, K.G.: Universality, marginal operators, and limit cycles. Phys. Rev. B69, 094304 (2004)

128. Gubankova, E.L., Wegner, F.J.: Flow equations for QED in the light front dynamics. Phys. Rev. D58, 025012 (1998)

129. Grote, I., Körding, E., Wegner, F.: Stability analysis of the Hubbard model. J. Low Temp. Phys. 126, 1385 (2002)

130. Halboth, C.J., Metzner, W.: Renormalization group analysis of the two-dimensional Hubbard model. Phys. Rev. B61, 7364 (2000)

131. Hankevych, V., Grote, I., Wegner, F.: Pomeranchuk and other instabilities in the t-t' Hubbard model at the Van Hove filling. Phys. Rev. B66, 094516 (2002)

132. Hofstetter, W., Kehrein, S.: Flow equation analysis of the anisotropic Kondo model. Phys. Rev. B63, 140402 (2001)

133. Jones, B.D., Perry, R., Głazek, S.D.: Nonperturbative QED: an analytical treatment on the light front. Phys. Rev. D55, 6561 (1997)

134. Kabel, A., Wegner, F.: Flow equations for hamiltonians: crossover from Luttinger to Landau-liquid behaviour in the n-orbital model. Z. Phys. B103, 555 (1997)

135. Kehrein, S.: Scaling and decoherence in the nonequilibrium Kondo model. Phys. Rev. Lett. 95, 056602 (2005)

136. Kehrein, S.: The flow equation approach to many-particle systems, Springer tracts in modern physics, 217 (2006)

137. Kehrein, S., Mielke, A.: Flow equations for the Anderson hamiltonian. J. Phys. A: Math. Gen. 27, 4259 (1994), Corr. 27, 5705 (1994)

138. Kehrein, S., Mielke, A.: On the spin-boson model with a sub-Ohmic bath. Phys. Lett. A219, 313 (1996)

139. Kehrein, S., Mielke, A.: Low temperature equilibrium correlation functions in dissipative quantum systems. Ann. Phys. (Berlin) 6, 90 (1997)

140. Kehrein, S., Mielke, A.: Diagonalization of system plus environment hamiltonians. J. Stat. Phys. 90,889 (1998)

141. Kehrein, S., Mielke, A., Neu, P.: Flow equations for the spin-boson problem. Z. Phys. B99, 269 (1996)

142. Knetter, C., Uhrig, G.S.: Perturbation theory by flow equations: dimerized and frustrated $S=1 / 2$ chain. Eur. Phys. J. B13, 209 (2000)

143. Kraemer, T., Mark, M., Waldburger, P., Danzl, J.G., Chin, C., Engeser, B., Lange, A.D., Pilch, K., Jaakkola, A., Nägerl, H.-C., Grimm, R.: Evidence for Efimov quantum states in an ultracold gas of caesium atoms. Nature 440, 315 (2006)

144. Landau, L.D.: The theory of a Fermi liquid. Sov. Phys. JETP 3, 920 (1956)

145. Landau, L.D.: Oscillations in a Fermi liquid. Sov. Phys. JETP 5, 101 (1957)

146. Landau, L.D.: On the theory of the Fermi liquid. Sov. Phys. JETP 8, 70 (1959)

147. Lenz, P., Wegner, F.: Flow equations for electron-phonon interactions. Nucl. Phys. B 482, 693 (1996)

148. Mielke, A.: Similarity renormalization of the electron-phonon coupling. Ann. Phys. (Berlin) 6, 215 (1997)

149. Mohr, R.F., Furnstahl, R.J., Perry, R.J., Wilson, K.G., Hammer, H.-W.: Precise numerical results for limit cycles in the quantum three-body problem. Ann. Phys. 321, 225 (2006)

150. Raas, C., Bühler, A., Uhrig, G.S.: Effective spin models for spin-phonon chains by flow equations. Eur. Phys. J. B21, 369 (2001)

151. Salmhofer, M., Honerkamp, C.: Fermionic renormalization group flow - technique and theory. Prog. Theor. Phys. 105, 1 (2001)

152. Schrieffer, J.R., Wolff, P.A.: Relation between the Anderson and Kondo hamiltonians. Phys. Rev. 149, 491 (1966)

153. Shankar, R.: Renormalization group for interacting fermions in $d>1$. Physica A177, 530 (1991)

154. Shankar, R.: Renormalization group approach to interacting fermions. Rev. Mod. Phys. 66, 129 (1994)

155. Thimmel, B.: Flussgleichungen für das Kondo-Modell, Diploma thesis, Heidelberg (1996)

156. Thomas, L.H.: The interaction between a neutron and a proton and the structure of $\mathrm{H}^{3}$. Phys. Rev. 47, 903 (1935)

157. Vogel, E.: Flussgleichungen für das Kondo-Modell, Diploma thesis, Heidelberg (1997)

158. Wegner, F.: Flow equations for Hamiltonians. Ann. Phys. (Berlin) 3, 77 (1994)

159. Wegner, F.: Flow equations and normal ordering: a survey. J. Phys. A: Math. Gen. 39, 8221 (2006) 
160. Wilson, K.G.: Model of coupling-constant renormalization. Phys. Rev. D2, 1438 (1970)

161. Wilson, K.G., Walhout, T.S., Hadrindranath, A., Zhang, W.M., Perry, R.J., Głazek, S.D.: A weakcoupling treatment on the light front. Phys. Rev. D49, 6720 (1994)

162. Zanchi, D., Schulz, H.J.: Weakly correlated electrons on a square lattice: renormalization group theory. Phys. Rev. B61, 13609 (2000) 